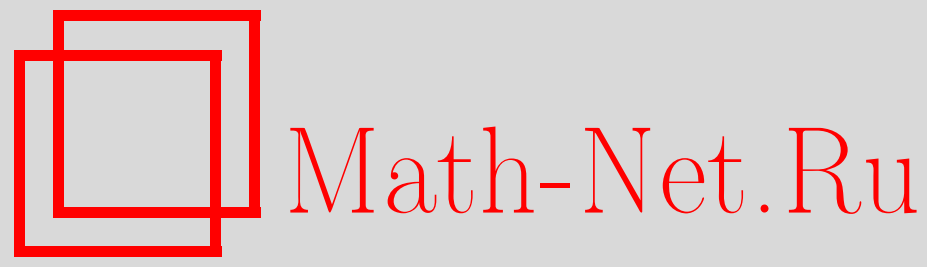

Р. С. Исмагилов, Ю. И. Любич, Кватернионное нормированное пространство с группой изометрий $\mathbb{Z}_{2}$, Функи. анализ и его прил., 2008, том 42, выпуск 3, 90-92

DOI: https://doi.org/10.4213/faa2917

Использование Общероссийского математического портала MathNet.Ru подразумевает, что вы прочитали и согласны с пользовательским соглашением http://www . mathnet.ru/rus/agreement

Параметры загрузки:

IP : 18.234 .197 .8

26 апреля 2023 г., 10:31:44

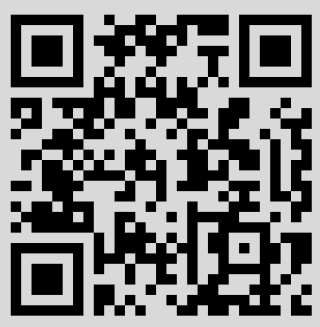


УДК 517.51

\title{
Кватернионное нормированное пространство с группой изометрий $\mathbb{Z}_{2}{ }^{*}$
}

\author{
(c) 2008. Р. С. ИсмАГИЛОВ, Ю. И. ЛЮБИч
}

Группа линейных изометрических преобразований вещественного линейного нормированного пространства может оказаться конечной; в случае комплексного пространства аналогичная группа всегда бесконечна, ибо любое преобразование вида $x \mapsto \alpha x$, где $\alpha$ - комплексное число и $|\alpha|=1$, изометрично. Рассмотрим теперь классическое тело кватернионов $\mathbb{H}$ и пространство $\mathbb{H}^{n}$, состоящее из всех столбцов $x=\left(x_{1}, \ldots, x_{n}\right)^{\prime}, x_{s} \in \mathbb{H}$. Введя в $\mathbb{H}^{n}$ умножение справа (для определенности) на элементы из $\mathbb{H}$, мы получаем (правое) $\mathbb{H}$-линейное пространство. Заметим, что в пространстве $\mathbb{H}^{n}$ умножение $x \mapsto x \lambda$ не является $\mathbb{H}$-линейным отображением, если $\lambda \notin \mathbb{R}$.

В докладе Ю. И. Любича (WONRA-6, Бремен, 15-17 июля 2006) была введена норма

$$
\|x\|=\left(\sum_{k=1}^{N}\left|x_{1}+\phi_{k}\left(x_{2}, \ldots, x_{n}\right)\right|^{p}\right)^{1 / p}, \quad x \in \mathbb{H}^{n},
$$

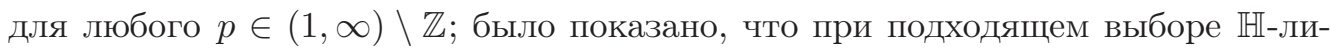
нейных функционалов $\phi_{k}$ группа $\mathbb{H}$-линейных изометрических (по отношению к норме (1)) преобразований пространства $\mathbb{H}^{n}$ конечна. В предлагаемой заметке мы несколько усовершенствуем выбор функционалов $\phi_{k}$; при таком выборе группа линейных изометрий сведется к $\mathbb{Z}_{2}=\{ \pm \mathrm{id}\}$.

Рассмотрим множество $\mathbb{Z}^{2}$; его элементы будут обозначаться через $(s, r)$. Возьмем целые числа $1=N_{1}<\cdots<N_{n}$ и рассмотрим подмножество $E \subset \mathbb{Z}^{2}$ вида $E=\left\{(s, r): 1 \leqslant s \leqslant n, 1 \leqslant r \leqslant N_{s}\right\}$. Для каждого $s=1, \ldots, n$ обозначим через $E_{s}$ множество точек $(s, r) \in E$ (с фиксированным $s$ ); назовем множества $E_{s}$ слоями. Возьмем, далее, ступенчатую кватернионную матрицу $\left(h_{s r}\right)$, $h_{s r} \in \mathbb{H}$, со следующими свойствами:

a) $h_{11}=0, h_{s r} \neq 0$ для всех $(s, r) \neq(1,1),(s, r) \in E$;

b) $\sum_{r=1}^{N_{s}} h_{s r}=0$ для всех $s \in\{2, \ldots, n\}$;

с) для любого $s \in\{2, \ldots, n\}$ величины $\left|h_{s r}\right|, r=1, \ldots, N_{s}$, попарно различны;

d) любой кватернион, перестановочный со всеми $h_{s r},(s, r) \in E$, есть вещественное число;

е) в любом слое $E_{s}, s=2, \ldots, n$, существует такая точка $(s, r)$, что $h_{s r}-$ вещественное число.

Используя эту матрицу, введем в $\mathbb{H}^{n}$ норму (разновидность нормы (1)) по формуле

$$
\|x\|=\left(\sum_{(s, r) \in E}\left|x_{1}+h_{s r} x_{s}\right|^{p}\right)^{1 / p} .
$$


Теорема. При выполнении условий а)-е) любая линейная изометрия пространства $\mathbb{H}^{n}$ по отношению $к$ норме (2) есть либо $x \mapsto x$, либо $x \mapsto-x$, $x \in \mathbb{H}^{n}$.

В доказательстве следующей леммы мы используем метод сравнения особенностей, развитый в работах [1]-[3].

Лемма 1. Пусть два семейства неотрицательных квадратичных форм $\left(f_{k}(x)\right)_{1}^{N} u\left(g_{k}(x)\right)_{1}^{N}$ удовлетворяют условию

$$
\sum_{k=1}^{N}\left(f_{k}(x)\right)^{\alpha}=\sum_{k=1}^{N}\left(g_{k}(x)\right)^{\alpha}, \quad x \in \mathbb{R}^{n},
$$

для некоторого $\alpha \in \mathbb{R}, \alpha>0,2 \alpha \notin \mathbb{Z}$. Пусть формы $f_{k}$ попарно не пропорииональны и тем же свойством обладают формы $g_{k}$. Тогда семейство $\left(g_{k}\right)_{1}^{N}$ получается перестановкой семейства $\left(f_{k}\right)_{1}^{N}$.

Доказательство. Обозначим через $V$ множество всех таких пар $(x, y)$, что $f_{k}(y)>0$ для всех $k \in\{1, \ldots, N\}, f_{k}(x) f_{l}(y)-f_{k}(y) f_{l}(x) \neq 0$ при $k \neq l$ и аналогичные условия выполнены для семейства $\left(g_{k}\right)_{1}^{N}$. Для любой точки $(x, y) \in V$ рассмотрим соотношение (3) на прямой $\{x+\tau y, \tau \in \mathbb{R}\}$, продолжим это соотношение аналитически на комплексные $\tau$ и изучим точки ветвления полученных слагаемых (корни квадратичных многочленов $f_{k}(x+\tau y), g_{k}(x+\tau y)$ ). Согласно выбору множества $V$, многочлены $f_{k}(x+\tau y)$ попарно не пропорциональны. Так как, кроме того, $2 \alpha \notin \mathbb{Z}$, то у функции в левой части равенства имеется $N$ различных пар точек ветвления (числа, входящие в пару, комплексно сопряжены; некоторые пары могут состоять из одинаковых вещественных чисел). То же самое имеет место и для функции в правой части равенства. Отсюда следует, что набор многочленов $\left\{g_{k}(x+\tau y)\right\}$ получается перестановкой набора $\left\{f_{m}(x+\tau y)\right\}$. В частности, многочлен $g_{1}(x+\tau y)$ совпадает с некоторым $f_{m}(x+\tau y)$ (номер $m$ зависит от $x, y)$, откуда $g_{1}(y)=f_{m}(y)$. Итак, для любого $y \in \mathbb{R}^{n}$, входящего в некоторую пару $(x, y) \in V$ (ясно, что множество таких векторов $y$ плотно в $\left.\mathbb{R}^{n}\right)$, существует такой номер $m=m(y)$, что $g_{1}(y)=f_{m}(y)$. Следовательно, для некоторого $m$ множество векторов $y$, удовлетворяющих условию $g_{1}(y)=f_{m}(y)$, плотно в некотором шаре. Но тогда это равенство выполнено для всех $y \in \mathbb{R}^{n}$. Итак, $g_{1}=f_{m}$. Продолжая это рассуждение, приходим к утверждению леммы.

Нам понадобится также следующий элементарный факт.

Лемма 2. Пусть строки $a=\left(a_{1}, \ldots, a_{n}\right), b=\left(b_{1}, \ldots, b_{n}\right), a_{r} \in \mathbb{H}, b_{r} \in \mathbb{H}$, удовлетворяют условию $|a x|=|b x|$ для всех $x \in \mathbb{H}^{n}$. Тогда $b=\theta a$ для некоторого $\theta \in \mathbb{H},|\theta|=1$.

Докажем теорему. Введем вектор-строки $e_{s}=\left(\delta_{s j}\right)_{j=1}^{n}, \delta_{s j}-$ символ Кронекера. Тогда можно чуть преобразовать формулу (2), переписав выражение $x_{1}+h_{s r} x_{s}$ из (2) в виде $\left(e_{1}+h_{s r} e_{s}\right) x$.

Обратимся теперь к $\mathbb{H}$-линейным изометриям пространства $\mathbb{H}^{n}$. Пусть $T=$ $\left(t_{s \sigma}\right), 1 \leqslant s, \sigma \leqslant n,-$ такая кватернионная матрица, что $\|x\|=\|T x\|, x \in \mathbb{H}^{n}$. Это равенство приводится к виду (3) при $\alpha=p / 2$; в роли квадратичных форм из (3) выступают $x \mapsto\left|\left(e_{1}+h_{s r} e_{s}\right) x\right|^{2}$ и $x \mapsto\left|\left(e_{1}+h_{s r} e_{s}\right) T x\right|^{2}$. Применяя леммы 1 и 2 , получаем такую биекцию $\phi: E \rightarrow E$ и такие элементы $\theta_{s r} \in \mathbb{H},(s, r) \in E$, что $\left|\theta_{s r}\right|=1$ и $e_{1} T+h_{s r} e_{s} T=\theta_{s r}\left(e_{1}+h_{s^{\prime} r^{\prime}} e_{s^{\prime}}\right)$, где $(s, r) \in E,\left(s^{\prime}, r^{\prime}\right)=\phi(s, r)$. 
Отсюда и из условия $h_{11}=0$ получаем равенства

$$
t_{11}+h_{s r} t_{s 1}=\theta_{s r}
$$

И

$$
t_{1 j}+h_{s r} t_{s j}=\theta_{s r} h_{s^{\prime} r^{\prime}} \delta_{s^{\prime} j}, \quad(s, r) \in E, j \in\{2, \ldots, n\} .
$$

Так как $h_{11}=0$, то из (4) следует, что $t_{11}=\theta_{11}$, а потому $\left|t_{11}\right|=1$. Отсюда и из (4) вытекает, что $\left|h_{s r}\right|^{2}\left|t_{s 1}\right|^{2}+t_{11} \bar{t}_{s 1} \bar{h}_{s r}+h_{s r} t_{s 1} \bar{t}_{11}=0,(s, r) \in E, s \geqslant 2$. Складывая эти равенства по слою $E_{s}$ и применяя свойства а) и b), приходим к равенствам $t_{s 1}=0$ при $s \geqslant 2$. Таким образом, равенство (4) сводится к $\theta_{s r}=t_{11}$ при $s \geqslant 2$. Учитывая это равенство, сложим равенства (5) по всем $(s, r) \in E$; применяя свойства a) и b), получаем, что $t_{1 j}=0, j \geqslant 2$. Теперь (5) принимает вид

$$
h_{s r} t_{s j}=t_{11} h_{s^{\prime} r^{\prime}} \delta_{s^{\prime} j}, \quad(s, r) \in E, j \geqslant 2,\left(s^{\prime}, r^{\prime}\right)=\phi(s, r) .
$$

Возьмем в этой формуле $(s, r)=(1,1)$ и положим $\phi(1,1)=(k, l)$. Тогда $t_{11} h_{k l} \delta_{k j}=0$; таким образом, $h_{k l}=0$ либо $\delta_{k j}=0$ для всех $j \geqslant 2$. В обоих случаях $k=1$ и $l=1$. Итак, биекция $\phi$ сохраняет слой $E_{1}$. Покажем, что и остальные слои инвариантны относительно $\phi$. Действительно, из (6) и условия а) вытекает, что $t_{s j}=0$ для всех $j \neq s^{\prime}$ и $t_{s s^{\prime}} \neq 0$. Следовательно, $s^{\prime}$ не зависит от $r$, а потому слой $E_{s}$ отображается в слой $E_{s^{\prime}}$. Так как количества элементов в $E_{2}, \ldots, E_{n}$ попарно различны, то эти слои инвариантны относительно $\phi$.

Теперь соотношение (6) принимает вид $h_{s r} t_{s j}=t_{11} h_{s r^{\prime}} \delta_{s j},(s, r) \in E, s \geqslant 2$, $j \geqslant 2,\left(s, r^{\prime}\right)=\phi(s, r)$. Отсюда, вспоминая равенства $t_{s 1}=t_{1 j}=0(s, j \geqslant 2)$, видим, что матрица $T$ диагональна. Далее, $\left|t_{s s}\right|=1$, ибо $T-$ изометрия. Теперь (6) сводится к равенству $h_{s r} t_{s s}=t_{11} h_{s r^{\prime}}$. Отсюда, используя свойство с), получаем равенство $r^{\prime}=r$. Далее, свойство е) показывает, что $t_{s s}=t_{11}$, а свойство d) дает $t_{11} \in \mathbb{R}$. Отсюда $t_{11}= \pm 1$ и, наконец, $T= \pm \mathrm{id}$. Теорема доказана.

В последнее время авторы рассмотрели нормы вида $\|x\|=\left(\sum_{k}\left|u_{k} x\right|^{p}\right)^{1 / p}$, где $p \in\{1, \infty\} \backslash \mathbb{Z}$ и $u_{k}=\left(u_{k 1}, \ldots, u_{k n}\right), u_{k j} \in \mathbb{H}, 1 \leqslant k \leqslant N, N>n+1$. Оказывается, что при выполнении некоторого полиномиального неравенства вида $P\left(\left\{u_{k j}\right\}\right) \neq 0$ любая линейная изометрия имеет вид $\pm \mathrm{id}$.

Замечание. Наше доказательство переносится на $\mathbb{R}$ и $\mathbb{C}$ и в обоих случаях дает $T=\mathrm{id} \cdot \lambda,|\lambda|=1$, даже без условий $\mathrm{d})$, e). Чтобы охватить все три случая $(\mathbb{R}, \mathbb{C}, \mathbb{H})$, достаточно добавить, что $\lambda$ принадлежит центру тела и соответственно изменить условие е). Однако условие $\mathrm{d}$ ) в такой общей формулировке следует опустить.

\section{ЛитеРАТУРА}

[1] Ю. И. Любич, Сиб. матем. ж., 11:2 (1970), 359-369.

[2] Ю. И. Любич, О. А. Шаталова, Алгебра и анализ, 16:1 (2004), 15-32. [3] Yu. I. Lyubich, L. N. Vaserstein, Geom. Dedicata, 47:3 (1993), 327-362.

Московский государственный технический университет им. Н. Э. Баумана e-mail: ismagil@serv.bmstu.ru Technion, Israel Institute of Technology e-mail: lyubich@techunix.technion.ac.il
Поступило в редакцию 27 сентября 2006 г. 